\title{
A complex gene regulatory architecture underlies the development and evolution of cuticle morphology in Drosophila
}

Sebastian Kittelmann ${ }^{1 *}$, Ella Preger-Ben Noon ${ }^{2 *}$, Alistair P. McGregor ${ }^{3}$ and Nicolás Frankel 4,5

${ }^{1}$ Centre for Functional Genomics, Department of Biological and Medical Sciences, Faculty of Health and Life Sciences, Oxford Brookes University, Oxford OX3 OBP, United Kingdom.

${ }^{2}$ Department of Genetics and Developmental Biology, The Rappaport Faculty of Medicine and Research Institute, Technion - Israel Institute of Technology, Haifa 3109601, Israel.

${ }^{3}$ Department of Biological and Medical Sciences, Faculty of Health and Life Sciences, Oxford Brookes University, Oxford OX3 OBP, United Kingdom.

${ }^{4}$ Instituto de Fisiología, Biología Molecular y Neurociencias (IFIBYNE, CONICET-UBA) and Departamento de Ecología, Genética y Evolución (FCEyN, UBA), Buenos Aires 1428, Argentina.

${ }^{5}$ Corresponding author: Nicolas Frankel <nfrankel@conicet.gov.ar>

* equal contribution

Keywords: morphological evolution, cuticle, trichome, shavenbaby, enhancer, gene regulatory network

Short title: Transcriptional regulation and morphological evolution 


\begin{abstract}
The cuticle of insects is decorated with non-sensory hairs called trichomes. A few Drosophila species independently lost most of the dorso-lateral trichomes on first instar larvae. Genetic experiments revealed that this naked cuticle phenotype was caused by the evolution of enhancer function at the ovo/shavenbaby (ovo/svb) locus. Here we explore how this discovery catalyzed major new insights into morphological evolution in different developmental contexts, enhancer pleiotropy in gene regulation and the functionality and evolution of the Svb gene regulatory network (GRN). Taken together this highlights the importance of understanding the architecture and evolution of gene regulatory networks in detail and the great potential for further study of the Svb GRN.
\end{abstract}




\section{Introduction}

The quest for understanding the genetic basis of morphological evolution has been at the forefront of evolutionary research for decades. The evolution of cuticle morphology in Drosophila larvae has proven to be a powerful model for helping to decipher the genetic and molecular mechanisms underlying morphological change. The apparent simplicity of these morphological transitions contrasts with the complex and fascinating picture that emerged at the genetic and molecular level. Here we discuss these findings, which have important implications for understanding the evolution of gene regulation and phenotypic diversity.

\section{The genetic basis underlying the evolution of larval morphology in Drosophila}

The cuticle of insects is decorated with non-sensory hairs, called microtrichia or trichomes. It has been proposed that trichomes enhance the hydrophobicity of the cuticle [1], reduce air resistance during flight or aid in larval locomotion [2]. In the genus Drosophila, firstinstar larvae exhibit a repetitive segmental pattern of trichomes (Figure 1A). In most Drosophila species the dorso-lateral cuticle is covered by a dense lawn of fine trichomes, called quaternary trichomes. Early work showed that the pattern of quaternary trichomes has evolved multiple times within the genus $[3,4]$. In $D$. sechellia, a species closely related to $D$. melanogaster, these trichomes were lost, causing a naked cuticle phenotype (Figure 1A) [4]. Similarly, species of the $D$. virilis species group exhibit both inter and intra-specific variation in quaternary trichome numbers $[3,5,6]$.

Genetic mapping experiments revealed that the naked cuticle phenotype of $D$. sechellia results entirely from the evolution of a single locus named ovo/shavenbaby (ovo/svb) [4]. This locus generates two transcripts in the germline (ovoA and ovoB) and 
one transcript in somatic cells (svb) [7]. "Hairy" species like D. melanogaster express svb mRNA in their epidermal quaternary cells, whereas "naked" species like $D$. sechellia lack $s v b$ expression in these cells (Figure 1A) [4,5]. The $s v b$ mRNA encodes a zinc-finger transcription factor that controls the expression of dozens of downstream effector genes that collectively promote trichome differentiation [8,9]. The Svb protein is synthesized with an $\mathrm{N}$-terminus that contains a transcriptional repression domain [5] and $\mathrm{N}$-terminal degradation by the proteasome converts Svb into a transcriptional activator [10]. This degradation step is mediated by small peptides encoded by the tarsal-less locus (tal, also known as polished-rice or mille-pattes) [11,12], whose expression is controlled by ecdysone signaling [13].

How did $D$. sechellia lose the expression of $s v b$ specifically in quaternary cells? The exhaustive dissection of the $\sim 100 \mathrm{~kb}$ region upstream of $s v b$ first exon in $D$. melanogaster revealed that seven enhancers control the complex epidermal expression of $s v b$ in late embryos (Figure 1B) [14,15]. These enhancers drive both unique and overlapping expression patterns, providing robustness to $s v b$ expression under environmental or genetic variation [15]. In $D$. sechellia, five of the $s v b$ enhancers evolved reduced activity specifically in embryonic quaternary cells $[14,15]$. Likewise, the convergent evolution of a naked dorso-lateral cuticle in $D$. ezoana, a species of the virilis species group, was caused by parallel genetic changes in orthologous $s v b$ enhancers [16].

One of the $s v b$ enhancers that evolved reduced activity in $D$. sechellia, named E6, was studied in detail to uncover the precise genetic and molecular mechanisms of enhancer evolution. E6 drives strong expression in quaternary cells of $D$. melanogaster embryos, but multiple genetic changes, each of small effect, reduced the activity of this enhancer in D. sechellia (Figure 1B) [17]. Six single-nucleotide substitutions and one single-nucleotide deletion led to the disruption of transcription factor binding sites (TFBSs) for transcriptional activators and, remarkably, to the creation of a novel TFBS for the transcriptional repressor Abrupt [18]. Both the fact that all mutations in the D. sechellia E6 enhancer diminish svb expression and the creation of a novel TFBS for a transcriptional repressor, suggest that the loss of trichomes in $D$. sechellia evolved by natural selection.

Originally, the function of the svb gene regulatory network (GRN) was described in the late Drosophila embryo. A further exploration of the activity of this GRN in later stages of Drosophila development uncovered important aspects of cis-regulation and GRN evolution. In addition, the characterization of $s v b$ homologs in distant species exposed a likely ancestral role for ovo/svb. 


\section{Pleiotropic enhancers and pleiotropic TFBSs}

It is often assumed that enhancers are tissue-specific regulatory elements and that TFBSs within enhancers are not reused in different contexts [19]. However, few studies have analyzed the activity of the same set of enhancers in different organs and/or developmental stages in detail. Therefore, the idea that enhancers are mostly active in unique tissues at specific times of development, as opposed to being active in multiple developmental contexts (i.e., being pleiotropic), remains hypothetical [19,20].

Contrary to the expectations, it was recently shown that the seven embryonic enhancers of $s v b$ also drive expression in many tissues during larval and pupal stages, demonstrating that these regulatory elements have a pleiotropic function (Figure 1C) $\left[21^{\star *}\right]$. In the same vein, genome-wide analyses suggest that pleiotropic enhancers are common in animal genomes [19]. This implies that small genomic fragments $(<1 \mathrm{~kb})$ might encode the regulatory information for driving expression in multiple developmental contexts. Remarkably, a recent screen that identified hundreds of transcriptional silencers in the embryonic mesoderm of $D$. melanogaster, showed that some of these repressive elements can also function as transcriptional enhancers in other contexts [22]. This means that there are not only pleiotropic enhancers in genomes, but pleiotropic regulatory elements that, depending on the context, can have distinct functional features [23].

Although there is growing evidence for the ubiquity of pleiotropic enhancers, there is very little knowledge regarding structural aspects of these regulatory elements. Are pleiotropic enhancers constituted by independent pieces of regulatory information (i.e., independent sets of TFBSs each driving a different expression pattern) that are spatially overlapped or closely juxtaposed? Alternatively, do pleiotropic enhancers re-use TFBSs for driving their multiple expression patterns? So far, the detailed analyses of enhancers from svb $\left[21^{* *}, 24^{* *}\right]$, scute [25] and yellow [26*] genes in Drosophila have shown that TFBS can be reused in different developmental contexts. However, additional enhancer dissections in multiple contexts are needed in order to determine whether site pleiotropy (i.e., TFBS reuse) is a common theme in the function of pleiotropic enhancers.

Another interesting issue that has been explored to some extent is how enhancers become pleiotropic [27]. For example, it has been shown that a large part of an ancestral Hox-regulated GRN was redeployed to make a novel structure in the male genitalia of Drosophila, and that this co-option event instantly turned the enhancers within the GRN into pleiotropic enhancers [28]. Also, a recent study showed how a single enhancer of the yellow gene, which drives an ancestral and broad expression pattern in the wing of 
Drosophila species, turned into a pleiotropic regulatory element in D. biarmipes [26*]. In this species, the use of ancestral regulatory information together with the acquisition of new TFBSs generated a novel and restricted expression pattern in the wing [26*].

Finally, a detailed study of the genetic changes underlying wing pigmentation differences in Heliconius butterflies has provided a glimpse on the evolution of pleiotropic enhancers [29]. This work suggests that pleiotropic enhancers of the optix gene with broad expression domains in the wings evolve through the gain or loss of repressor TFBSs rather than through changes in activator TFBSs [29,30].

\section{The topology of the svb GRN in the larva and leg of Drosophila and the evolution of GRNs}

The pivotal role of Svb and the architecture of the GRN that controls the development of larval trichomes is understood in great detail $\left[8,9,31,32^{* *}\right]$. However, trichomes also develop on the cuticle of adult structures, opening the question as to whether and how the GRN differs in these diverse developmental contexts. This has been explored by studying the development and patterning of trichomes on the second (T2) pair of adult legs [32**-35]. As in larvae, Svb plays an important role in determining which epidermal cells of the adult produce trichomes $\left[21^{* *}, 32^{* *}, 35\right]$. Moreover, most of the other genes with a characterized role in the Svb GRN in larvae are also expressed in developing legs $\left[32^{\star *}\right]$, implying that there are broad similarities between the larval and leg GRNs. However, there are also a number of key differences which have been analyzed using a trichome-free region on the proximal posterior T2 femur (the so-called naked valley). It is likely that the ancestral state in $D$. melanogaster is a large trichome-free area (a large naked valley) and that a reduction of the size of the naked valley (a gain of trichomes) evolved within $D$. melanogaster $[32,34]$. Genetic mapping between $D$. melanogaster strains with contrasting phenotypes in the naked valley revealed that this variation is not due to regulatory changes in $s v b$, but instead is caused by changes in the expression of a microRNA named miR-92a [34]. This microRNA does not appear to be involved in larval trichome patterning, but it blocks trichome formation on legs by post-transcriptionally repressing at least two Svb target genes, shavenoid and CG14395 [32**,34]. A second difference in the GRN is related to the function of the Hox gene Ultrabithorax (Ubx), which activates the formation of larval trichomes in D. melanogaster [31], but represses the formation of leg trichomes $\left[32^{\star *}, 33,35\right]$. Furthermore, it appears that Ubx-mediated 
repression of leg trichomes depends on miR-92a, suggesting that Ubx may directly activate the expression of this microRNA in legs [32**,35].

Comparisons between larval and leg trichome regulation have provided key insights into GRN structure and evolution, suggesting that the nodes of a GRN that evolve to generate phenotypic change may be context dependent, although further comparisons are needed to explore the generality of this finding. For example, changes in the regulation of scute and tartan genes in Drosophila contribute to polygenic interspecific changes in bristle numbers on the hypandrium and surstyli (male genital structures) respectively [25,36], which suggests that different genes have evolved in these two contexts. However, exactly the same change in scute also underlies variation in the number of sex comb bristles in T1 male legs [25]. Therefore, it will be interesting to describe these GRNs in greater detail and to identify the other genes therein that contribute to these morphological differences.

\section{An ancient GRN involved in cell differentiation}

Svb activates the expression of more than 150 target genes that promote trichome growth in Drosophila [9]. It appears that Svb function in the epidermis and some of the downstream components of this GRN are conserved in other flies [37], wasps [38*], beetles $\left[38^{*}, 39\right]$, hemipterans $\left[38^{*}, 40\right]$ and even in the more distantly related crustacean Daphnia [41](Figure 2).

In addition, the proteolytic processing of Svb via Tal is a key step required for Svb function in contexts other than the epidermis throughout insects (Figure 2). Indeed, svb and tal have a role in the formation of leg joints in Drosophila and other insects [38,42], as well as a function in insect segmentation, which was lost in flies [38]. Moreover, it has been shown that the homeostasis and differentiation of renal and intestinal stem cells in Drosophila are also regulated by $s v b$ and tal $[43,44]$. It is likely that Svb interacts with distinct partners in the many contexts in which it is active, with the concomitant activation of different sets of target genes. For example, Svb partners with the transcription factor Yki in renal stem cells [43], while in the embryonic epidermis Svb cooperates with the transcription factor SoxN [45].

Genes homologous to ovo/svb are found throughout metazoans and are called OVO-like (OVOL) in other clades [46]. In mice OVOL1 is involved in the formation of epidermal hairs and spermatogenesis [47] and OVOL2 is required for germline development, which resembles the functions of ovo/svb in trichome formation and 
germline development in Drosophila [48]. In addition, OVOL1 and OVOL2 are required in vertebrates for epithelial maintenance by suppressing the epithelial-mesenchymal transition [49-52]. Interestingly, it has been shown that Svb interacts with epithelialmesenchymal transition promoting factors during stem cell homeostasis in Drosophila $[43,44]$. Thus, it is likely that a Svb-like protein had a role in germline development and epithelial differentiation/tissue homeostasis in the urbilaterian ancestor (Figure 2), although studies in more organisms are required to confirm this hypothesis.

\section{Concluding remarks}

Studies of the evolution of larval morphology in Drosophila uncovered the genetic and molecular causes of phenotypic evolution at unprecedented resolution. An exhaustive analysis of the $s v b$ gene facilitated the identification of several transcriptional enhancers, allowing for the study of enhancer function in the context of a whole cis-regulatory region and for a precise and quantitative measure of the effect of genetic changes during enhancer evolution. At the same time, the analysis of trichome pattern variation in the Drosophila leg and the dissection of the svb GRN in the leg showed that the preferred evolutionary paths might be context dependent. Altogether, these studies have advanced the knowledge on the genetic bases of morphological evolution. Nonetheless, it is clear that we still have a superficial understanding of cis-regulatory logic and GRN evolution. Undoubtedly, the next decades of shavenbaby research will provide, again, with important insights on the evolution of transcriptional regulation.

\section{Acknowledgements}

We thank François Payre and an anonymous reviewer for helpful comments on the manuscript, and David L. Stern, the initiator of shavenbaby evolutionary research, for his early insight and continuous enthusiasm.

\section{Author contributions}

SK, APM, EPBN and NF conceived the idea of the article and wrote the manuscript. 


\section{Figure captions}

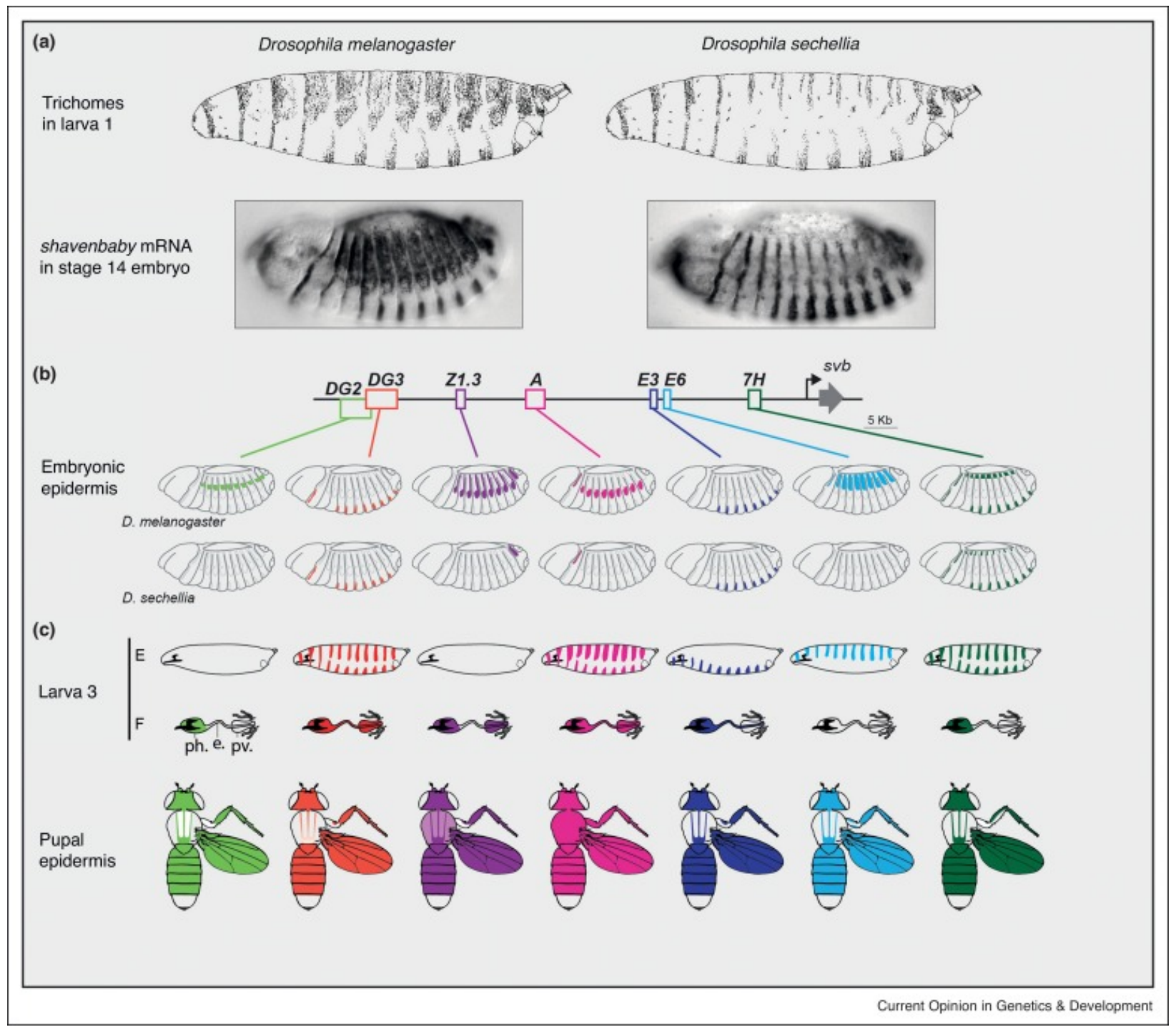

Figure 1. Larval trichome patterns have evolved between Drosophila species through changes in the cis-regulatory region of the ovo/shavenbaby (ovo/svb) gene. (A) Top: Drawing of trichome patterns in lateral views of first instar larvae of $D$. melanogaster (left) and its closely related species $D$. sechellia (right). Bottom: svb mRNA patterns in stage 14 embryos of $D$. melanogaster (left) and D. sechellia (right). Adapted from McGregor et al., 2007 [14]. (B) Structure of the $s v b$ locus, indicating the position of embryonic enhancers (colored boxes) and their respective expression pattern in the embryonic epidermis of $D$. melanogaster (top) and $D$. sechellia (bottom). svb coding region is marked in gray. (C) Representation of the expression patterns driven by the $D$. melanogaster svb enhancers in 
the third instar larva epidermis (top) and foregut (middle), and in the pupal epidermis (bottom). Ph: pharynx, e: esophagus, pv: proventriculus.

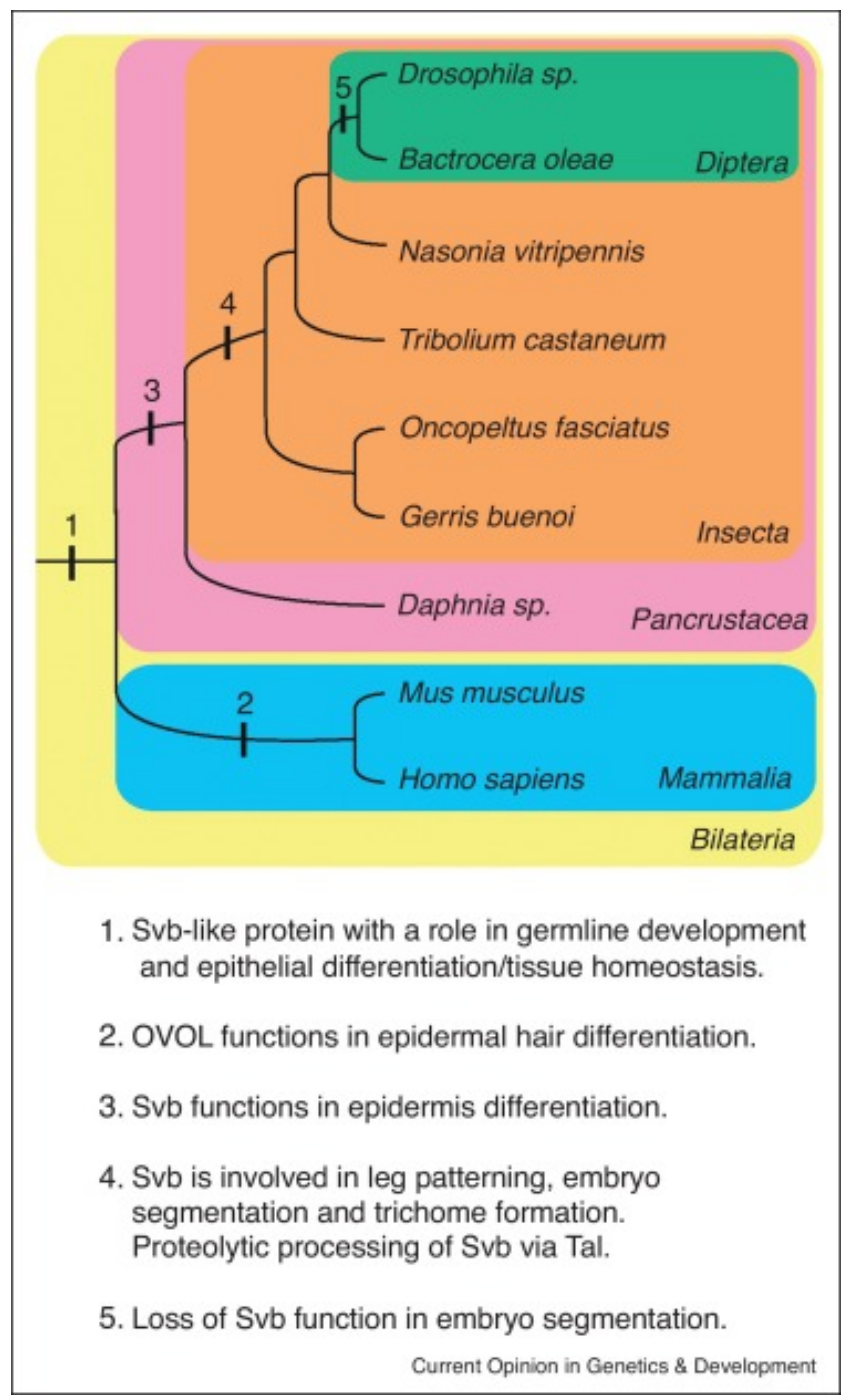

Figure 2. The evolution of the function of ovo/svb genes. Based on current literature, we propose a hypothesis for the evolution of the different functions of ovo/svb genes in distant linages. The species that are shown in the phylogenetic tree are the ones for which there is information on ovo/svb function. Numbers in branches indicate likely events in relation to the evolution of the function of ovo/svb genes (see text below). 


\section{Declaration of interests}

The authors declare no conflict of interest

\section{References and recommended reading}

Fuqua et al. $2020[\cdot]$ In this work, the authors performed a mutagenesis of $s v b E 3 N$ enhancer in $D$. melanogaster, identifying nucleotide sites that are needed for proper embryonic expression. This study reveals that many TBFSs within E3N are pleiotropic and that regulatory information is densely packed in a small DNA region.

Xin et al. 2020 [·] This paper shows that an ancestral enhancer of the yellow gene became pleiotropic by being co-opted for a new function in the wing of Drosophila biarmipes. This novel function relies on old TFBSs as wells as newly acquired TFBSs.

Ray et al. 2019 [.] By studying the role of $s v b$ in several species this paper demonstrates that $s v b$ and tal have ancestral functions in embryo segmentation, leg patterning and cuticle formation in insects. This paper also shows that the segmentation function of $s v b$ was lost in D. melanogaster.

Preger-Ben Noon et al. 2018 [*] This article shows that the svb gene is expressed in multiple tissues of the larva and pupa of $D$. melanogaster and that $s v b$ expression in the pupal epidermis is necessary for trichome formation in adult structures. This work proves that $s v b$ embryonic enhancers are also active during larval and pupal stages and that the E6 enhancer uses the same TFBSs to drive expression in both the embryonic epidermis and pupal epidermis.

Kittelmann et al. 2018 [.] By analyzing the svb GRN in the developing leg of $D$. melanogaster, the authors identified key differences in the svb GRN between the adult leg and the embryo epidermis. These differences reveal why mutations in $s v b$ are unlikely to contribute to leg trichome variation.

1. van Breugel F, Dickinson MH: Superhydrophobic diving flies (Ephydra hians ) and the hypersaline waters of Mono Lake. Proceedings of the National Academy of Sciences 2017, 114:13483-13488. 
2. Inestrosa NC, Sunkel CE, Arriagada J, Garrido J, Godoy-Herrera R: Abnormal development of the locomotor activity in yellow larvae of Drosophila: a cuticular defect? Genetica 1996, 97:205-210.

3. Dickinson WJ, Yang Y, Schuske K, Akam M: Conservation of Molecular Prepatterns during the Evolution of Cuticle Morphology in Drosophila Larvae. Evolution 1993, 47:1396.

4. Sucena E, Stern DL: Divergence of larval morphology between Drosophila sechellia and its sibling species caused by cis-regulatory evolution of ovo/shaven-baby.

Proceedings of the National Academy of Sciences of the United States of America 2000, 97:4530-4.

5. Sucena E, Delon I, Jones I, Payre F, Stern DL: Regulatory evolution of shavenbaby/ovo underlies multiple cases of morphological parallelism. Nature 2003, 424:935-8.

6. Soverna AF, Rodriguez NC, Korgaonkar A, Hasson E, Stern DL, Frankel N: Cisregulatory variation in the shavenbaby gene underlies intraspecific phenotypic variation, mirroring interspecific divergence in the same trait. Evolution 2021, doi:https://doi.org/10.1111/evo.14142.

7. Mével-Ninio M, Terracol R, Salles C, Vincent A, Payre F: ovo, a Drosophila gene required for ovarian development, is specifically expressed in the germline and shares most of its coding sequences with shavenbaby, a gene involved in embryo patterning. Mechanisms of Development 1995, 49:83-95.

8. Chanut-Delalande H, Fernandes I, Roch F, Payre F, Plaza S: Shavenbaby couples patterning to epidermal cell shape control. PLoS biology 2006, 4:e290.

9. Menoret D, Santolini M, Fernandes I, Spokony R, Zanet J, Gonzalez I, Latapie Y, Ferrer $\mathrm{P}$, Rouault $\mathrm{H}$, White KP, et al.: Genome-wide analyses of Shavenbaby target genes reveals distinct features of enhancer organization. Genome biology 2013, 14:R86.

10. Zanet J, Benrabah E, Li T, Pelissier-Monier A, Chanut-Delalande H, Ronsin B, Bellen HJ, Payre F, Plaza S: Pri sORF peptides induce selective proteasome-mediated protein processing. Science 2015, 349:1356-1358.

11. Kondo T, Plaza S, Zanet J, Benrabah E, Valenti P, Hashimoto Y, Kobayashi S, Payre F, Kageyama Y: Small peptides switch the transcriptional activity of Shavenbaby during Drosophila embryogenesis. Science (New York, NY) 2010, 329:336-9.

12. Kondo T, Hashimoto Y, Kato K, Inagaki S, Hayashi S, Kageyama Y: Small peptide regulators of actin-based cell morphogenesis encoded by a polycistronic mRNA. Nature Cell Biology 2007, 9:660-665.

13. Chanut-Delalande H, Hashimoto Y, Pelissier-Monier A, Spokony R, Dib A, Kondo T, Bohère J, Niimi K, Latapie Y, Inagaki S, et al.: Pri peptides are mediators of ecdysone for the temporal control of development. Nature cell biology 2014, 16:1035-44.

14. McGregor AP, Orgogozo V, Delon I, Zanet J, Srinivasan DG, Payre F, Stern DL: Morphological evolution through multiple cis-regulatory mutations at a single gene. Nature 2007, 448:587-90.

15. Frankel N, Davis GK, Vargas D, Wang S, Payre F, Stern DL: Phenotypic robustness conferred by apparently redundant transcriptional enhancers. Nature 2010, 466:490-493.

16. Frankel N, Wang S, Stern DL: Conserved regulatory architecture underlies parallel genetic changes and convergent phenotypic evolution. Proceedings of the National Academy of Sciences 2012, 109:20975-20979.

17. Frankel N, Erezyilmaz DF, McGregor AP, Wang S, Payre F, Stern DL: Morphological evolution caused by many subtle-effect substitutions in regulatory DNA. Nature 2011, 474:598-603.

18. Preger-Ben Noon E, Davis FP, Stern DL: Evolved Repression Overcomes Enhancer 
Robustness. Developmental cell 2016, 39:572-584.

19. Sabarís G, Laiker I, Preger-Ben Noon E, Frankel N: Actors with Multiple Roles:

Pleiotropic Enhancers and the Paradigm of Enhancer Modularity. Trends in Genetics 2019, 35:423-433.

20. Monteiro A, Podlaha O: Wings, Horns, and Butterfly Eyespots: How Do Complex Traits Evolve? PLoS Biology 2009, 7:e1000037.

21. Preger-Ben Noon E, Sabarís G, Ortiz D, Sager J, Liebowitz A, Stern DL: Comprehensive analysis of a cis-regulatory region reveals pleiotropy in enhancer function. Cell reports 2018, 22:3021-3031.

22. Gisselbrecht SS, Palagi A, Kurland J V., Rogers JM, Ozadam H, Zhan Y, Dekker J, Bulyk ML: Transcriptional Silencers in Drosophila Serve a Dual Role as Transcriptional Enhancers in Alternate Cellular Contexts. Molecular Cell 2020, 77:324-337.e8.

23. Halfon MS: Silencers, Enhancers, and the Multifunctional Regulatory Genome. Trends in Genetics 2020, 36:149-151.

24. Fuqua T, Jordan J, van Breugel ME, Halavatyi A, Tischer C, Polidoro P, Abe N, Tsai A, Mann RS, Stern DL, et al.: Dense and pleiotropic regulatory information in a developmental enhancer. Nature 2020, 587:235-239.

25. Nagy O, Nuez I, Savisaar R, Peluffo AE, Yassin A, Lang M, Stern DL, Matute DR, David JR, Courtier-Orgogozo V: Correlated Evolution of Two Copulatory Organs via a Single cis-Regulatory Nucleotide Change. Current Biology 2018, 28:3450-3457.e13.

26. Xin Y, Le Poul Y, Ling L, Museridze M, Mühling B, Jaenichen R, Osipova E, Gompel N: Ancestral and derived transcriptional enhancers share regulatory sequence and a pleiotropic site affecting chromatin accessibility. Proceedings of the National Academy of Sciences 2020, 117:20636-20644.

27. Rice G, Rebeiz M: Evolution: How Many Phenotypes Do Regulatory Mutations Affect? Current Biology 2019, 29.

28. Glassford WJ, Johnson WC, Dall NR, Smith SJ, Liu Y, Boll W, Noll M, Rebeiz M: Cooption of an Ancestral Hox-Regulated Network Underlies a Recently Evolved Morphological Novelty. Developmental Cell 2015, 34:520-531.

29. Lewis JJ, Geltman RC, Pollak PC, Rondem KE, Van Belleghem SM, Hubisz MJ, Munn PR, Zhang L, Benson C, Mazo-Vargas A, et al.: Parallel evolution of ancient, pleiotropic enhancers underlies butterfly wing pattern mimicry. Proceedings of the National Academy of Sciences 2019, 116:24174-24183.

30. Lewis JJ, Van Belleghem SM: Mechanisms of Change: A Population-Based Perspective on the Roles of Modularity and Pleiotropy in Diversification. Frontiers in Ecology and Evolution 2020, 8:261.

31. Crocker J, Abe N, Rinaldi L, McGregor AP, Frankel N, Wang S, Alsawadi A, Valenti P, Plaza S, Payre F, et al.: Low affinity binding site clusters confer hox specificity and regulatory robustness. Cell 2015, 160:191-203.

32. Kittelmann S, Buffry AD, Franke FA, Almudi I, Yoth M, Sabaris G, Couso JP, Nunes MDS, Frankel N, Gómez-Skarmeta JL, et al.: Gene regulatory network architecture in different developmental contexts influences the genetic basis of morphological evolution. PLOS Genetics 2018, 14:e1007375.

33. Stern DL: A role of Ultrabithorax in morphological differences between Drosophila species. Nature 1998, 396:463-466.

34. Arif S, Murat S, Almudi I, Nunes MDS, Bortolamiol-Becet D, McGregor NS, Currie JMS, Hughes H, Ronshaugen M, Sucena É, et al.: Evolution of mir-92a Underlies Natural Morphological Variation in Drosophila melanogaster. Current Biology 2013, 23:523528.

35. Buffry AD, Kittelmann S, McGregor AP: Characterisation of the role and regulation of \&lt;em\&gt;Ultrabithorax\&lt;/em\&gt; in sculpting fine-scale leg morphology. bioRxiv 
2020, doi:10.1101/2020.06.17.152918.

36. Hagen JFD, Mendes CC, Blogg A, Payne A, Tanaka KM, Gaspar P, Figueras Jimenez J, Kittelmann M, McGregor AP, Nunes MDS: tartan underlies the evolution of Drosophila male genital morphology. Proceedings of the National Academy of Sciences 2019, 116:19025-19030.

37. Khila A, EI Haidani A, Vincent A, Payre F, Souda SI: The dual function of ovo/shavenbaby in germline and epidermis differentiation is conserved between Drosophila melanogaster and the olive fruit fly Bactrocera oleae. Insect Biochemistry and Molecular Biology 2003, 33:691-699.

38. Ray S, Rosenberg MI, Chanut-Delalande H, Decaras A, Schwertner B, Toubiana W, Auman T, Schnellhammer I, Teuscher M, Valenti P, et al.: The mlpt/Ubr3/Svb module comprises an ancient developmental switch for embryonic patterning. eLife 2019, 8.

39. Li C, Yun X, Li B: Dusky-like is required for epidermal pigmentation and metamorphosis in Tribolium castaneum. Scientific Reports 2016, 6:20102.

40. Tobias-Santos V, Guerra-Almeida D, Mury F, Ribeiro L, Berni M, Araujo H, Logullo C, Feitosa NM, de Souza-Menezes J, Pessoa Costa E, et al.: Multiple Roles of the Polycistronic Gene Tarsal-less/Mille-Pattes/Polished-Rice During Embryogenesis of the Kissing Bug Rhodnius prolixus. Frontiers in Ecology and Evolution 2019, 7:379.

41. Spanier KI, Jansen M, Decaestecker E, Hulselmans G, Becker D, Colbourne JK, Orsini L, De Meester L, Aerts S: Conserved Transcription Factors Steer Growth-Related Genomic Programs in Daphnia. Genome Biology and Evolution 2017, 9:1821-1842.

42. Pueyo JI, Couso JP: Tarsal-less peptides control Notch signalling through the Shavenbaby transcription factor. Developmental biology 2011, 355:183-93.

43. Bohère $J$, Mancheno-Ferris $A, A$ I Hayek $S$, Zanet $J$, Valenti $P$, Akino $K$, Yamabe $Y$, Inagaki S, Chanut-Delalande H, Plaza S, et al.: Shavenbaby and Yorkie mediate Hippo signaling to protect adult stem cells from apoptosis. Nature Communications 2018, 9:5123.

44. Al Hayek S, Alsawadi A, Kambris Z, Boquete JP, Bohère J, Ronsin B, Plaza S, Lemaitre $B$, Payre F, Osman D: Shavenbaby protein isoforms orchestrate the self-renewal versus differentiation of Drosophila intestinal stem cells. bioRxiv 2019, doi:10.1101/627554.

45. Rizzo NP, Bejsovec A: SoxNeuro and Shavenbaby act cooperatively to shape denticles in the embryonic epidermis of Drosophila. Development 2017, 144:22482258.

46. Kumar A, Bhandari A, Sinha R, Sardar P, Sushma M, Goyal P, Goswami C, Grapputo A: Molecular Phylogeny of OVOL Genes Illustrates a Conserved C2H2 Zinc Finger Domain Coupled by Hypervariable Unstructured Regions. PLoS ONE 2012, 7:e39399.

47. Dai X, Schonbaum C, Degenstein L, Bai W, Mahowald A, Fuchs E: The ovo gene required for cuticle formation and oogenesis in flies is involved in hair formation and spermatogenesis in mice. Genes \& Development 1998, 12:3452-3463.

48. Hayashi M, Shinozuka Y, Shigenobu S, Sato M, Sugimoto M, Ito S, Abe K, Kobayashi S: Conserved role of Ovo in germline development in mouse and Drosophila. Scientific Reports 2017, 7:40056.

49. Jia D, Jolly MK, Boareto M, Parsana P, Mooney SM, Pienta KJ, Levine H, Ben-Jacob E: OVOL guides the epithelial-hybrid-mesenchymal transition. Oncotarget 2015, 6:15436-15448.

50. Li S, Yang J: Ovol Proteins: Guardians against EMT during Epithelial Differentiation. Developmental Cell 2014, 29:1-2.

51. Nieto MA, Huang RY-J, Jackson RA, Thiery JP: EMT: 2016. Cell 2016, 166:21-45.

52. Saxena K, Srikrishnan S, Celia-Terrassa T, Jolly MK: OVOL1/2: Drivers of Epithelial 
Differentiation in Development, Disease, and Reprogramming. Cells Tissues Organs 2020, doi:10.1159/000511383. 